\title{
Adverse neonatal outcomes and associated factors among mothers who gave birth through cesarean section at Arba Minch General Hospital, Southern Ethiopia
}

\author{
Damtie Getie ${ }^{1}$, Befikadu Tariku Gutema ${ }^{2}$, William Haleke ${ }^{3}$, Nega Degefa Megersa ${ }^{4}$ and \\ Kassahun Fikadu Tessem ${ }^{5}$
}

\author{
${ }^{1}$ Shebel Berenta Primary Hospital, East Gojjam, Amhara Regional State, Ethiopia. \\ ${ }^{2}$ Public Health Department, College of Medicine and Health Sciences, Arba Minch University, Arba Minch, Ethiopia. \\ ${ }^{3}$ School of Medicine, College of Medicine and Health Sciences, Arba Minch University, Arba Minch, Ethiopia. \\ ${ }^{4}$ Department of Nursing, College of Medicine and Health Sciences, Arba Minch University Arba Minch, Ethiopia. \\ ${ }^{5}$ Department of Midwifery, College of Medicine and Health Sciences, Arba Minch University, Arba Minch, Ethiopia.
}

Received 24 July, 2019; Accepted 28 March, 2020

Cesarean section (CS) refers to the delivery of fetus, placenta, and membrane through an abdominal and uterine incision after 28 completed weeks of gestation. This mode of delivery minimizes both maternal and perinatal morbidity and mortality; however, it is associated with lower but severe neonatal outcomes as compared to vaginal delivery. Thus, the current study was aimed to assess the current status of adverse neonatal outcomes and its contributing factors. A retrospective cross-sectional study was employed from July 2016 to August 2017. The data were collected from records of mothers who gave birth through CS in Arba Minch General Hospital using a structured record review checklist. Simple random sampling technique was used to select records for extracting data. Variables with pvalue $<0.25$ in the bivariate model were entered into multivariable model to declare statistical significance at $p$-value $<0.05$. Results revealed that nearly one-third of newborns delivered through cesarean section developed adverse outcome. Rural residency (AOR: 2.96; 95\% Cl: 1.47, 5.96), fetus with cephalopelvic disproportion (AOR: 2.58 ; $95 \% \mathrm{Cl}: 1.23,5.42$ ), CS with general anesthesia (AOR 3.28, 95\% Cl: 1.21, 8.93) and maternal complication due to CS (AOR: 2.38 ; 95\% Cl: 1.21, 4.70) were determinants of adverse neonatal outcome. Newborns delivered at term, have lower risk of adverse outcome as compared to pre-term (AOR: $0.11 ; 95 \% \mathrm{Cl}: 0.01,0.93$ ). Mothers with prolonged rupture of membrane before CS was 3.33 times more likely to deliver a baby with adverse outcome than their counterparts (AOR: $3.33 ; 95 \% \mathrm{Cl}: 1.13,9.84$ ). It can therefore be concluded that nearly one-third of newborns delivered through cesarean section developed an adverse outcome. Each caesarian delivery with cephalopelvic disproportion, pre-term caesarian delivery, and under general anesthesia should get prompt and appropraite management. 


\section{INTRODUCTION}

Cesarean section delivery signifies the expulsion of a fetus, placenta, and membrane through abdominal and uterine incision after 28 completed weeks of gestation (Alan et al., 2007). This mode of delivery has played significant role in curtailing maternal and perinatal morbidity and mortality in the past century. Initially, the procedure was performed to save the life of mothers with dystocia, and over years indications have extended to include delivery for a variety of more mild dangers to the mother or fetus. Evidence suggests that, frequent use of cesarean delivery is mainly due to increased safety of cesarean section that is mostly the result of improved surgical technique, better use of anesthesia, effective antibiotics, and accessibility of blood for transfusion (Cunningham et al., 2005; Menacker and Hamilton, 2010).

In the United States, cesarean-section delivery increased by $53 \%$ from 1996 to 2007, getting 32\%, which is reported the highest rate ever (Menacker and Hamilton, 2010). In the year 2006 in America, nearly onethird (31.1\%) of all births were cesarean deliveries. The increase in cesarean delivery includes both primary and repeat cesarean. In addition, some studies noted that, the risk of neonatal and maternal mortality increases for medically elective cesareans when compared with vaginal birth (Signore et al., 2006; Wax, 2006; MacDorman et al., 2008).

Evidences suggest that, cesarean section delivery may be mandatory when vaginal delivery impose a risk either to the mother or the baby due to prolonged labor, fetal distress, or abnormal presentation (Hannah et al., 2000). In fact, emergency cesarean delivery was accompanied with more fresh stillbirths, neonatal deaths and severe neonatal morbidity probably related to prolonged labor, asphyxia, and sepsis (Shah et al., 2009).

The World Health Organization (WHO) statement published currently declares that, when cesarean section rates rise towards $10 \%$ across a population, the amount of maternal and newborn deaths decreases. However, there is no evidence that suggests improvement in the mortality rate with an increasing cesarean rate beyond $10 \%$. Furthermore, across a population, the effects of cesarean section rates on maternal and newborn outcomes such as stillbirths or morbidities like birth asphyxia still remain unknown (Organization, 2015).

Three-quarters of all women from hospitals of subSaharan Africa gave birth through CS mainly for maternal reasons which include: prolonged labor, abruptio placenta, earlier cesarean section, eclampsia, placenta previa, and mal-presentation (Dumont et al., 2001).

According, to 2008 Ethiopian Ministry of Health Report, overall, even with the fertility decline, the population is still growing at an annual rate of $2.6 \%$ (Health, 2010). Increase in the rate of cesarean delivery was associated with an increase in fetal mortality rates and admission to neonatal intensive care unit for seven or more days. An increase in the rate of cesarean delivery between 10$20 \%$, raises the rate of both preterm delivery and neonatal mortality (José Villar et al, 2006). Compared with a plan for vaginal delivery, cesarean delivery on maternal request may be associated with a lower rate but severe neonatal outcomes (Ecker, 2013).

Arba Minch General Hospital is a center of Comprehensive Emergency Obstetric Care (CEmOC). Hence, there is a higher prevalence of cesarean section delivery which was $24.65 \%$ with emergency cesarean delivery accounting for $87 \%$ of all cesarean deliveries. Fetal distress, Cephalopelvic Disproportion (CPD) and an obstructed labor accounted for half of all indications for cesarean section (Wae et al., 2017). There were plenty of studies that compare the outcomes of delivery by mode of delivery yet, there is dearth of evidence on adverse neonatal outcomes among mothers who gave birth through cesarean section. Thus, the current study was aimed to assess, adverse neonatal outcomes and associated factors among mothers who gave birth through a cesarean section in Arba Minch General Hospital.

\section{MATERIALS AND METHODS}

\section{Study setting}

The study was conducted at Arba-Minch General Hospital (AGH) in Arba Minch Town. Arba Minch town is located in Gamo Gofa Zone of the southern nations, nationalities and peoples region about 505 $\mathrm{km}$ south of Addis Ababa. Arba-Minch General Hospital is one of the governmental hospitals in Gamo Zone of Southern Ethiopia.

\section{Study design and period}

A retrospective cross-sectional study design was employed. The data was collected in December 2017 from selected records of mothers who gave birth through CS from July 2016 to August 2017.

\section{Sample size and sampling procedure}

A single population proportion formula was used to calculate the sample size by considering the following assumption: $95 \%$ level of certainty, $5 \%$ margin of error, $22 \%$ proportion of neonates with the adverse outcome from the previous study in Ethiopia (Yenit et al., 2016) and $10 \%$ for incomplete record. Thus, the total sample size was 290 records. The list of mothers who gave birth through cesarean-section from July 2016 to August 2017 in the hospital was

*Corresponding author. Email: negshd05@gmail.com or nega.degefu@amu.edu.et 
identified by referring to the delivery room, operation room, and postnatal ward log books. Identification number of mothers who gave birth through CS from July 2016 to August 2017 was obtained from the record. Using Stata version 14, table of random numbers were generated to identify the Identification number of mothers selected for review.

\section{Eligibility criteria}

All recorded women who gave birth by cesarean section at Arba Minch General Hospital from July 2016 to August 2017 were included in the study. Records of mothers with ectopic pregnancy and incomplete records were excluded.

\section{Data collection}

A structured and pre-tested record review checklist was used to collect data from delivery records The English version of the record review checklist was adapted and developed from similar studies conducted previously (Moges et al., 2015; Yenit et al., 2016; Wae et al., 2017). Selected records were collected from the respective units to extract the necessary variables using their identification number.

\section{Variables and definition}

The dependent variable was an adverse neonatal outcome following cesarean birth. The independent variables include maternal socio-demographic characteristics, pregnancy-related characteristics, delivery-related factors, and maternal health service utilization related characteristics.

Adverse neonatal outcome: These were considered when the neonate has one of the following: low APGAR scores, hypoglycemia, low birth weight, pre-term birth, neonatal asphyxia, sepsis, meconium aspiration syndrome or neonatal seizures.

Maternal CS delivery complication: Intra-operative surgical complications that include damage to adjacent organs during surgery, incision extension, hemorrhage, atony or blood transfusion during operation.

Obstetric complications: These refer to the presence of one of the following condition; Antepartum hemorrhage (APH), PROM/ chorioamnionitis, preeclampsia or eclampsia, obstructed labor, malpresentation, suspected uterine rupture, previous cesarean delivery or gynecology operations.

\section{Quality control}

To ensure the quality of data, a pre-test was done on $5 \%$ of the sample. A two days training was given for data collectors and supervisor on data collection. In addition, the collected data were checked for completeness, accuracy, and consistency on a daily basis.

\section{Data processing and analysis}

The collected data were coded, cleaned and entered using Epidata version 3.1 and analyzed using Stata version 14. Descriptive statistics were carried out to explain the socio-demographic characteristics and magnitude of neonatal outcomes following cesarean section. Bivariate and multivariable logistic regression was done and odds ratio with $95 \%$ confidence interval was used to identify the presence and strength of association between variables. Independent variables which showed association with the dependent variable in the bivariate analysis at $\mathrm{P}<0.25$ were included in the final model for multivariable analysis. Multicollinearity was checked using variance inflation factor and standard error. Variables with $p$-value less than 0.05 was considered significant in all cases. Model fitness was plaid using Hosmer- Lemeshow goodness of fit test.

\section{Ethical clearance}

Letter of ethical clearance was obtained from Arba Minch University Research and ethical review board. Official letter from the university was submitted to Arba Minch General Hospital to obtain their cooperation. Since we extracted secondary data, consent for participation was not applicable.

\section{RESULTS}

From the total selected records of mothers, $286(98.6 \%)$ were reviewed. Four (1.6\%) were excluded due to incomplete recording. The mean age of mothers who gave birth by CS was 26.19 ( \pm SD5.29) years and the majority (65.4\%) were less than 30 years old. Nearly $55 \%$ of all mothers were referred from other health facilities. About, half of the mothers had four and above ANC visits whereas, 9.4\% had no visit at all (Table 1).

Nearly one-third, 90 (31.8\%) of neonates developed adverse outcome following cesarean delivery. From the total record reviewed, $2(0.7 \%)$ neonates die immediately after birth. From alive newborn, 33 (11.6\%) were admitted to the neonatal intensive care unit (NICU), the leading causes of admission were: perinatal asphyxia (57.6\%), low birth weight (15.2\%), sepsis (12.1\%) and preterm birth (12.1\%) and cause of admission for one newborn was unrecorded. Out of the total admitted neonates, 7 (21.2\%) died and 26 (78.8\%) had good progress at discharge (Table 2).

From the total reviewed records, 29 (10.1\%) of all mothers had a medical illness during pregnancy; hypertension and diabetes mellitus accounts for the biggest proportion. Only $44(15 \%)$ of the mothers underwent CS before the onset of labor. Cephalopelvic disproportion was the leading cause of neonatal complication in $27.3 \%$ of all mothers who delivered through CS (Table 3).

Age of the mother, residency, referral from other health facilitates, ANC visit, type of CS, type of anesthesia, maternal CS complication and obstetric complication, fetal distress, membrane status and duration of rapture, labor status and stage, cephalopelvic disproportion, obstructed labor, and gestational age were associated with adverse neonatal outcome on bivariate logistic regression.

The result of multivariable logistic regression analysis showed that residency, membrane status and duration, 
Table 1. Socio-demographic and cesarean delivery related characteristics of mothers $(n=286)$ who gave birth through CS categorized by neonatal complication Southern Ethiopia, 2017.

\begin{tabular}{|c|c|c|c|c|}
\hline \multirow{2}{*}{ Variable } & \multirow{2}{*}{ Category } & No Complication & Complication & Total \\
\hline & & \multicolumn{3}{|c|}{ [n (\%)] } \\
\hline \multirow{2}{*}{ Age of the mother } & $<30$ & 154(36.2) & $59(13.8)$ & $213(74.5)$ \\
\hline & $\geq 30$ & $41(28.1)$ & $32(21.9)$ & $73(25.5)$ \\
\hline \multirow{2}{*}{ Residency } & Urban & 107(39.9) & $27(10.1)$ & $134(46.9)$ \\
\hline & Rural & $88(28.9)$ & $64(21.1)$ & $152(53.1)$ \\
\hline \multirow{2}{*}{ Referred } & Yes & $94(29.9)$ & $63(20.1)$ & $157(54.9)$ \\
\hline & No & 101(39.1) & 28(10.9) & $129(45.1)$ \\
\hline \multirow{3}{*}{ Number of ANC visit } & 0 & $13(24.1)$ & $14(25.9)$ & $27(9.4)$ \\
\hline & $1-3$ & $79(32.6)$ & $42(17.4)$ & $121(42.3)$ \\
\hline & $\geq 4$ & 103(37.3) & $35(12.7)$ & $138(48.3)$ \\
\hline \multirow{2}{*}{ Maternal medical illness } & Yes & $19(32.8)$ & $10(17.2)$ & 29(10.10) \\
\hline & No & $176(34.2)$ & $81(15.8)$ & $257(89.9)$ \\
\hline \multirow{2}{*}{ Type of CS } & Emergency & 172(33) & $89(17.0)$ & 261(91.3) \\
\hline & Elective & $23(46)$ & $2(4.0)$ & $25(8.70)$ \\
\hline \multirow{2}{*}{ Type of anesthesia } & Spinal & $184(35.5)$ & $75(14.5)$ & $259(90.6)$ \\
\hline & General & $11(20.4)$ & $16(29.6)$ & $27(9.4)$ \\
\hline \multirow{2}{*}{ Duration of operation } & $<60 \min$ & 172(34.1) & $80(15.9)$ & 252(88.1) \\
\hline & $60-90 \mathrm{~min}$ & $23(33.8)$ & $11(16.2)$ & $34(11.9)$ \\
\hline \multirow{2}{*}{ Maternal CS delivery complication } & No & 151(38.1) & $47(11.9)$ & 198(69.2) \\
\hline & Yes & $44(25.0)$ & $44(25.0)$ & $88(30.8)$ \\
\hline \multirow{2}{*}{ Obstetric complication } & Yes & 118(34.1) & $55(15.9)$ & 173(60.5) \\
\hline & No & $77(34.1)$ & $36(15.9)$ & 113(39.5) \\
\hline
\end{tabular}

Table 2. Neonatal outcomes $(n=284)$ after cesarean section delivery in Arba Minch General Hospital, Southern, Ethiopia, 2017.

\begin{tabular}{lccc}
\hline Variable & Category & Frequency $(\mathbf{n})$ & $\%$ \\
\hline \multirow{3}{*}{ APGAR Score at $1^{\text {st }}$ minute } & $\leq 3$ & 2 & 0.70 \\
& $4-6$ & 97 & 34.15 \\
\multirow{2}{*}{ APGAR Score at the $5^{\text {th }}$ minute } & $\geq 7$ & 185 & 65.14 \\
& $4-6$ & 29 & 10.21 \\
& $\geq 7$ & 255 & 89.79 \\
\multirow{2}{*}{ Birth Weight (n=286) } & Low & 14 & 4.90 \\
& Normal & 233 & 81.47 \\
\multirow{2}{*}{ Neonatal resuscitation } & Large & 39 & 13.64 \\
\hline \multirow{2}{*}{ Prenatal asphyxia } & Yes & 82 & 28.87 \\
& No & 202 & 71.13 \\
\hline \multirow{3}{*}{ Neonatal sepsis } & Yes & 19 & 6.69 \\
& No & 265 & 93.31 \\
\hline
\end{tabular}

the presence of cephalopelvic disproportion, type of anesthesia, maternal CS delivery complication and gestational age had a significant association with adverse neonatal outcome. Hence, those mothers who came from rural areas were 2.96 times more likely to have adverse neonatal outcome compared with urban (AOR: 2.96; 95\% 
Table 3. Delivery related characteristics categorized by neonatal outcome after CS at Arba Minch General Hospital, Ethiopia, 2017.

\begin{tabular}{|c|c|c|c|c|}
\hline \multirow{2}{*}{ Variable } & \multirow{2}{*}{ Category } & No-complication & Complication & Total \\
\hline & & \multicolumn{3}{|c|}{ [n (\%)] } \\
\hline \multirow{2}{*}{ Fetal distress } & Yes & $63(30.6)$ & 40(19.4) & $103(36.0)$ \\
\hline & No & $132(36.1)$ & $51(13.9)$ & $183(64.0)$ \\
\hline \multirow{3}{*}{ Membrane status and duration } & Not ruptured & $74(39.4)$ & $20(10.6)$ & $94(32.9)$ \\
\hline & $\leq 12 \mathrm{~h}$ & 101(33.9) & $48(16.1)$ & $149(52.1)$ \\
\hline & $>12 \mathrm{~h}$ & $20(23.3)$ & $23(26.7)$ & $43(15.0)$ \\
\hline \multirow{2}{*}{ Duration of labor } & $\leq 24 \mathrm{~h}$ & 152(33.9) & $72(16.1)$ & $224(78.3)$ \\
\hline & $>24 \mathrm{~h}$ & 7(19.4) & 11(30.6) & $18(6.3)$ \\
\hline \multirow{4}{*}{ Labor status and stage } & No labor & $36(40.9)$ & $8(9.1)$ & $44(15.4)$ \\
\hline & Latent first stage & $65(36.1)$ & $25(13.9)$ & $90(31.5)$ \\
\hline & Active first stage & $73(31.7)$ & $42(18.3)$ & $115(40.2)$ \\
\hline & Second stage & $21(28.4)$ & $16(21.6)$ & $37(12.9)$ \\
\hline \multirow{4}{*}{ Cervical status } & Closed & $36(40.9)$ & $8(9.1)$ & $44(15.4)$ \\
\hline & $<4 \mathrm{~cm}$ & $65(36.1)$ & $25(13.9)$ & $90(31.5)$ \\
\hline & $4-9 \mathrm{~cm}$ & $73(31.7)$ & $42(18.3)$ & $115(40.2)$ \\
\hline & $10 \mathrm{~cm}$ & $21(28.4)$ & $16(21.6)$ & $37(12.9)$ \\
\hline \multirow{2}{*}{ Cephalopelvic disproportion } & No & $62(3$ for 9.7$)$ & $16(10.3)$ & $78(27.3)$ \\
\hline & Yes & 133(32) & $75(18.0)$ & $208(72.7)$ \\
\hline \multirow{2}{*}{ Mal presentation } & Yes & $20(40.0)$ & $5(10.0)$ & $25(8.7)$ \\
\hline & No & $175(33.5)$ & $86(16.5)$ & 261(91.3) \\
\hline \multirow{2}{*}{ Obstructed labor } & Yes & $5(16.7)$ & 10(33.3) & $15(5.2)$ \\
\hline & No & $190(35.1)$ & $81(14.9)$ & $271(94.8)$ \\
\hline \multirow{3}{*}{ Gestational age } & $<37$ & $2(11.1)$ & $7(38.9)$ & $9(3.1)$ \\
\hline & $37-42$ & 184(35.1) & $78(14.9)$ & $262(91.6)$ \\
\hline & $>42$ & $9(30.0)$ & $6(20.0)$ & $15(5.2)$ \\
\hline \multirow{2}{*}{ Number of pregnancy outcome } & Singleton & $179(33.6)$ & $87(16.4)$ & 266(93.0) \\
\hline & Twin & $16(40.0)$ & $4(10.0)$ & $20(7.0)$ \\
\hline
\end{tabular}

$\mathrm{Cl}: 1.47,5.96)$. The proportion of neonatal complication was lower for term born infant compared with pre-term (AOR: 0.11; 95\% Cl: 0.01, 0.93). A fetus with cephalopelvic disproportion was 2.58 times more likely to have an adverse neonatal complication (AOR: 2.58; 95\% $\mathrm{Cl}: 1.23,5.42)$.

Mothers with rupture of the membrane that lasted for more than 12-h before CS was 3.33 times more likely to give birth to a neonate with complication than those with a membrane not ruptured. (AOR: $3.33 ; 95 \% \mathrm{Cl}: 1.13$, 9.84). Mothers who underwent CS with general anesthesia had greater odds of having neonates with the adverse outcome as compared with spinal anesthesia (AOR: 3.28, 95\% Cl: 1.21, 8.93). Maternal complication due to CS delivery had significantly increased the proportion of neonatal complication by more than two times (AOR: 2.38; 95\% Cl: 1.21, 4.70) (Table 4).

\section{DISCUSSION}

The current study showed that, $31.8 \%$ of newborns delivered through cesarean section developed a adverse outcome. Perinatal asphyxia was the leading adverse neonatal outcome which lead to admission. A consistent finding was reported on two studies conducted in Ethiopia, at Finote Selam Hospital, Northwest and Attat Hospital, Central Ethiopia, which showed perinatal or birth asphyxia was the leading cause of admission to (NICU) (Moges et al., 2015; Yenit et al., 2016).

Our finding revealed that $11.6 \%$ of the live-born infants require immediate admission to NICU. which is slightly higher than the finding of a study at the University of Colorado Denver, USA which showed that $9.3 \%$ were admitted to NICU. However, this USA study was conducted to examine the outcome of neonates born by elective repeated cesarean delivery as compared with vaginal birth after cesarean delivery (Kamath et al., 2009).

In this study, infants born with increased gestational age were less likely to develop an adverse neonatal outcome. A study conducted to explore adverse neonatal outcome among elective caesarian delivery in America indicated similar finding and reported that infants 
Table 4. Factors associated with adverse neonatal outcome among mothers who gave birth through the cesarean section from July 2016 to August 2017 in Arba Minch General Hospital, Arba Minch, Ethiopia, 2017.

\begin{tabular}{|c|c|c|c|}
\hline Variable & Category & COR & AOR 95\%(Cl) \\
\hline \multirow{2}{*}{ Residency } & Urban & Ref & Ref \\
\hline & Rural & $2.88^{\star \star}$ & $2.96(1.47,5.96)$ ** \\
\hline \multirow{2}{*}{ Age of the mother (years) } & $<30$ & Ref & Ref \\
\hline & $\geq 30$ & $2.04^{\star}$ & $1.69(0.82,3.49)$ \\
\hline \multirow{2}{*}{ Referred } & Yes & Ref & Ref \\
\hline & No & $0.41^{\star *}$ & $0.67(0.33,1.36)$ \\
\hline \multirow{3}{*}{ Member status and duration } & Not ruptured & Ref & Ref \\
\hline & $\leq 12 \mathrm{~h}$ & 1.76 & $1.08(0.43,2.75)$ \\
\hline & $>12 \mathrm{~h}$ & $4.25^{\star \star}$ & $3.33(1.13,9.84)^{\star}$ \\
\hline \multirow{4}{*}{ Labor status and stage } & No labor & Ref & Ref \\
\hline & Latent first stage & 1.73 & $2.97(0.75,11.71)$ \\
\hline & Active first stage & $2.59^{\star}$ & $3.44(0.81,14.54)$ \\
\hline & Second stage & $3.43^{*}$ & $5.27(1.03,26.99)^{\star}$ \\
\hline \multirow{2}{*}{ Cephalopelvic disproportion } & No & Ref & Ref \\
\hline & Yes & $2.19^{*}$ & $2.58(1.23,5.42)^{\star}$ \\
\hline \multirow{2}{*}{ Mal-presentation } & Yes & Ref & Ref \\
\hline & No & 1.97 & $1.37(0.37,5.10)$ \\
\hline \multirow{2}{*}{ Obstructed labor } & Yes & Ref & Ref \\
\hline & No & $0.21^{\star \star}$ & $0.67(0.17,2.59)$ \\
\hline \multirow{2}{*}{ Type of anesthesia } & Spinal & Ref & Ref \\
\hline & General & $3.57^{\star *}$ & $3.28(1.21,8.93)^{\star}$ \\
\hline \multirow{3}{*}{ ANC follow up } & none & Ref & Ref \\
\hline & $1-3$ & 0.49 & $0.68(0.24,1.90)$ \\
\hline & $\geq 4$ & $0.32^{\star *}$ & $0.99(0.35,2.86)$ \\
\hline \multirow{2}{*}{ Maternal CS delivery complication } & No & Ref & Ref \\
\hline & Yes & $3.21^{\star *}$ & $2.38(1.21,4.70)^{\star}$ \\
\hline \multirow{2}{*}{ Type of CS } & Emergency & Ref & Ref \\
\hline & Elective & $0.17^{\star}$ & $0.52(0.08,3.48)$ \\
\hline \multirow{3}{*}{ Gestational age } & $<37$ weeks & Ref & Ref \\
\hline & 37- 42 weeks & $0.12^{\star *}$ & $0.11(0.01,0.93)^{\star}$ \\
\hline & $\geq 42$ weeks & 0.19 & $0.58(0.05,6.38)$ \\
\hline \multirow{2}{*}{ Number of pregnancy outcome } & Singleton & Ref & Ref \\
\hline & Twin & 0.51 & $0.54(0.14,2.01)$ \\
\hline
\end{tabular}

* P-Value $<0.05,{ }^{* *} \mathrm{P}-$ Value $<0.01$

delivered at 37-39 weeks encounter more serious outcome than those delivered above 39 weeks of gestation (Clark et al., 2009). Similaly, another observational study in Saudi Arabia also showed that lower gestational age was associated with adverse neonatal outcome (Alzahrani, 2017).

A study conducted by Sengupta et al. (2013) reported that early-term neonates were at higher risks for the development of hypoglycemia, NICU admission, need for respiratory support, and need for mechanical ventilation than term neonates (Sengupta et al., 2013). A similar finding was also reported by Bastek et al. (2008) which showed, late preterm infants, have increased the risk of adverse neonatal outcomes as compared with term infants. It indicated that with controlling for confounders, there was a $23 \%$ decrease in adverse outcomes with each week of advancing gestational age (Bastek et al., 2008).

The current study showed that babies born to mothers who took spinal anesthesia were less likely to have adverse neonatal outcomes including better Apgar score. A similar finding was reported in a study by Jawad Zahir et al. (2011), which showed that babies born to mothers who underwent spinal anesthesia had a significantly better one minute Apgar score. The study also reported that NICU admission was significantly higher in babies 
born with general anesthesia (28\%) as compared to babies born under spinal anesthesia (0.6\%) (Zahir et al., 2011).

The main strength of this study was the application of the proper model for analysis and sampling approach. However this study had also limitation like: the finding depends on the secondary source, which is subject to incomplete recording, missed values, and inconsistencies. Cross-sectional nature of the study was another limitation that made it difficult to establish a causal relationship.

\section{CONCLUSION AND RECOMMENDATION}

Health outcomes of cesarean delivery for both the mother and fetus have been bothersome. Nearly one-third of a newborn delivered through cesarean section develop adverse neonatal outcome which may result in admission to NICU or death. Mothers with preterm labor, cephalopelvic disproportion, CS complication, living in rural area, who encounter early rupture of the membrane prior to the cesarean section and under general anesthesia had an increased risk of having a baby with adverse outcome. The leading adverse neonatal outcome that contributes to the largest rate of admission to $\mathrm{NICU}$ was perinatal asphyxia. It is recommended that each cesarean delivery with cephalopelvic disproportion, preterm cesarean delivery, and use of general anesthesia should get due attention to take timely and appropriate measure, which can save the lives of the mother as well the fetus.

\section{ABBREVIATIONS}

ANC, Antenatal care; PNC, postnatal care; EDHS, Ethiopian Demographic and Health Survey; WHO, World Health Organization; AOR, adjusted odds ratio; $\mathbf{C l}$, confidence interval; NICU, neonatal intensive care unit, CS, cesarean section.

\section{CONFLICT OF INTERESTS}

The authors have not declared any conflict of interests.

\section{ACKNOWLEDGEMENTS}

The authors are grateful to Arba Minch University College of Medicine and Health Sciences for being open-handed to carry out this research project. Authors are pleased and appreciate the effort of data collectors, supervisors and the Medical Director of Arba Minch General Hospital for their unreserved support during data collection.

\section{REFERENCES}

Alan H, Decherney M, Lauren M, Nathan M, Murphy G, NuriLaufer M (2007). "Current Diagnosis \& Treatment Obstetrics \& Gynecology."
United States of America.

Alzahrani AK (2017). "Neonatal Outcomes in Relation to Timing of Term Cesarean Delivery: AnObservational Study." Journal of Neonatal and Pediatric Medicine 3(2).

Bastek JA, MD, Sammel E, Paré SK Srinivas M, Posencheg A, Elovitz MA (2008). "Adverse neonatal outcomes: examining the risks between preterm, late preterm, and term infants." American journal of obstetrics and gynecology 199(4):367-e1.

Clark SL, Miller DD, Belfort MA, Dildy GA, Frye DK, Meyers JA (2009). "Neonatal and maternal outcomes associated with elective term delivery." American journal of obstetrics and gynecology 200(2):156e1.

Cunningham FG, MacDonald PC, Gant NF (2005). Williams obstetrics, McGraw-Hill Professional New York, NY.

Dumont A, De Bernis L, Bouvier-olle MH, Bréart G, Group MS (2001). "Caesarean section rate for maternal indication in sub-Saharan Africa: a systematic review." The Lancet 358(9290):1328-1333.

Ecker J (2013). "Elective Cesarean Delivery on Maternal Request." jama 309(18).

Hannah ME, Hannah WJ, Hewson SA, Hodnett ED, Saigal S, Willan AR, Collaborative TBT (2000). "Planned caesarean section versus planned vaginal birth for breech presentation at term: a randomised multicentre trial." The Lancet 356(9239):1375-1383.

Health FDRoE Mo (2010). Health Sector Development Programme IV 2010/11-2014/15.

José Villar EV, Daniel Wojdyla Nelly Zavaleta, Guillermo Carroli A (2006). "Caesarean delivery rates and pregnancy outcomes: the 2005 WHO global survey on maternal and perinatal health in Latin America." Lancet 367:1819-1829.

Kamath BD, Todd JK, Glazner JE, Lezotte D, Lynch AM (2009). "Neonatal outcomes after elective cesarean delivery." Obstetrics and gynecology 113(6):1231.

MacDorman MF, Menacker F, Declercq E (2008). "Cesarean birth in the United States: epidemiology, trends, and outcomes." Clinics in perinatology 35(2): 293-307.

Menacker F, Hamilton BE (2010). Recent trends in cesarean delivery in the United States, US Department of Health and Human Services, Centers for Disease Control and Prevention, National Center for Health Statistics.

Moges A, Ademe BGJA, Akessa M (2015). "Prevalence and outcome of caesarean section in Attat Hospital, Gurage Zone, SNNPR, Ethiopia." 7(4):1-6.

Organization WH (2015). "WHO statement on caesarean section rates. 2015." Geneva, Switzerland: WHO.

Sengupta S, Carrion V, Shelton J, Wynn RJ, Ryan RM, Singhal K, Lakshminrusimha S (2013). "Adverse neonatal outcomes associated with early-term birth." JAMA pediatrics 167(11):1053-1059.

Shah A, Fawole B, M'Imunya JM, Amokrane F, Nafiou I, Wolomby JJ, Mugerwa K, Neves I, Nguti R, Kublickas M, (2009). "Cesarean delivery outcomes from the WHO global survey on maternal and perinatal health in Africa." International Journal of Gynecology \& Obstetrics 107(3):191-197.

Signore C, Hemachandra A, Klebanoff M (2006). Neonatal mortality and morbidity after elective cesarean delivery versus routine expectant management: a decision analysis. Seminars in perinatology, Elsevier.

Wae MB, Belayneh F, Mekonnen N, Hailemicheal F (2017). "Magnitude of maternal complications and associated obstetric factors among women who gave birth by cesarean section at Arba-Minich General Hospital, Southern Ethiopia: Retrospective cohort."

Wax JR (2006). Maternal request cesarean versus planned spontaneous vaginal delivery: maternal morbidity and short term outcomes. Seminars in perinatology, Elsevier.

Yenit MK, Gezahegn T, Adefires M, Shiferaw AMJGJoMR (2016). "Cesarean Section Rate, Maternal and Fetal Outcome of Birth Following Cesarean Section at Finoteselam Hospital, Northwest Ethiopia: A Descriptive Retrospective Data."

Zahir J, Syed S, Jabeen N, Anjum Q, Rehman S (2011). "Maternal and neonatal outcome after spinal versus general anaesthesia for caesarean delivery." Annual Pak Instruction Medical Science 7(3):115-118. 